\title{
Birational Classification of Curves on Irrational Ruled Surfaces
}

\author{
Osamu MATSUDA \\ Tsuyama National College of Technology \\ (Communicated by T. Kawasaki)
}

\section{Introduction.}

Let $S$ be a non-singular ruled surface with positive irregularity $q$ and $D$ an irreducible curve on $S$, which are defined over the field of complex numbers.

The purpose of this paper is to study pairs $(S, D)$ from the view point of birational geometry. Two pairs $(S, D)$ and $\left(S_{1}, D_{1}\right)$ are said to be birationally equivalent if there exists a birational map $\varphi: S \rightarrow S_{1}$ such that the proper image $\varphi[D]$ of $D$ by $\varphi$ coincides with $D_{1}$. Such $\varphi$ is said to be a birational transformation between pairs.

The pair $(S, D)$ is said to be non-singular whenever both $S$ and $D$ are non-singular. In this case, define $P_{m}[D]$ to be $\operatorname{dim} H^{0}(S, \mathcal{O}(m(D+K)))(m>0)$ and $\kappa[D]$ to be the $K+D$ dimension of $S$, which is denoted by $\kappa(D+K, S)$, where $K$ indicates a canonical divisor on $S$. Both $P_{m}[D]$ and $\kappa[D]$ are invariant under birational transformations between pairs. If every exceptional curve $E$ of the first kind on $S$ satisfies the inequality $E \cdot D \geq 2(E \neq D)$, then $(S, D)$ is said to be relatively minimal (cf. [I1], [Sa1]). Moreover, $(S, D)$ is said to be minimal, if every birational map from any non-singular pair $\left(S_{1}, D_{1}\right)$ into $(S, D)$ turns out to be a morphism. It is easily shown that every minimal pair is relatively minimal. Since $S$ is an irrational ruled surface, the Albanese map $\alpha: S \rightarrow \operatorname{Alb}(S)$ gives rise to a surjective morphism $\alpha: S \rightarrow \alpha(S)=B$, which is a curve of genus $q$. Let $F$ denote a general fiber of $\alpha: S \rightarrow B$. Then the intersection number $D \cdot F$ coincides with the mapping degree of $\left.\alpha\right|_{D}: D \rightarrow B$, which is denoted by $\sigma(D)$.

Every irrational ruled surface is obtained from a $\mathbf{P}^{1}$-bundle over $B$ by successive blowing ups. Suppose that $X$ is a $\mathbf{P}^{1}$-bundle and $C$ a curve on $X$. Then the group $\operatorname{Num}(X)$ of numerical equivalent classes of divisors on $X$ is a free abelian group generated by an infinite section $\Gamma_{\infty}$ and a fiber $F_{u}=\Phi^{-1}(u)$ of the $\mathbf{P}^{1}$-bundle $X$ where $\Phi$ is the projection (cf. [Ha], p. 370, Proposition 2.3). Then $C \equiv \sigma \Gamma_{\infty}+e F_{u}$ for some integers $\sigma$ and $e$ where the symbol $\equiv$ means numerical equivalence between divisors. Note that $\sigma=C \cdot F_{u}=\sigma(C)$. Let $b=-\Gamma_{\infty}^{2}$, which is said to be the degree of $X$. Moreover, let the multiplicities of all the singular points of $C$ be denoted by $m_{1}, m_{2}, \cdots, m_{r}\left(m_{1} \geq m_{2} \geq \cdots \geq m_{r}\right)$ where infinitely near singular points are 
included. Then the type of a pair $(X, C)$ is said to be $\left[\sigma * e, X ; m_{1}, m_{2}, \cdots, m_{r}\right]$. In the case where $C$ is itself non-singular, we put $r=1$ and $m_{1}=1$ by convention.

First, we give a birational classification of relatively minimal pairs $(S, D)$ as follows.

THEOREM 1. Suppose that $q=1$. Then we have the following table.

\begin{tabular}{c|c|c|c|l}
\hline class & $\kappa[D]$ & types & $g(D)$ & $P_{m}[D](m \geq 1)$ \\
\hline \hline \multirow{2}{*}{$\mathrm{I}$} & $-\infty$ & $\sigma(D)=0$ or 1 & 0 or 1 & 0 \\
\hline \multirow{2}{*}{$\mathrm{II}$} & 0 & {$\left[2 *-1, A_{-1} ; 1\right]$} & 1 & 1 (where $m$ is even) \\
\cline { 3 - 5 } & & {$\left[2 * 0, \Sigma_{(2)} ; 1\right]$} & 1 & $0($ where $m$ is odd $)$ \\
\hline \multirow{2}{*}{$\mathrm{II} \frac{1}{2}$} & \multirow{2}{*}{1} & {$\left[\sigma * 0, \Sigma_{(\sigma)} ; 1\right]$} & 1 & $1+2 s-m,(s=[m(\sigma-1) / \sigma])$ \\
\cline { 3 - 5 } & & {$\left[4 *-2, A_{-1} ; 1\right]$} & 1 & $1+3 s-m,(s=[m / 2])$ \\
\cline { 3 - 5 } & & {$[2 * e, X ; 1]$} & $e-b+1 \geq 2$ & $m(e-b)$ \\
\hline III & 2 & & $\geq 1$ & $\geq 2$ if $m \geq 2$. \\
\hline
\end{tabular}

Here, the surfaces $\Sigma_{(2)}, \Sigma_{(\sigma)}$ and $A_{-1}$ are elliptic surfaces where $\sigma \geq 3 . g(D)$ denotes the genus of a curve $D$ and the symbol $[x]$ denotes the integral part of a number $x$. The degree of $A_{-1}$ is -1 and the degrees of $\Sigma_{(2)}$ and $\Sigma_{(\sigma)}$ are zero.

Define $n$ to be $4 g(D)-D^{2}-8 q+4$.

THEOREM 2. Suppose that $q=1$ and that $(S, D)$ is a relatively minimal pair with $\kappa[D]=2$. Then $n \geq 1$ and $\sigma(D) \leq n(n+2)$. If $n=1$, then $\sigma(D)=3$. In this case, there exists a pair $(S, D)$ satisfying these conditions.

Now, the pair $(S, D)$ is said to be an Enriques pair if the type is either $\left[2 *-1, A_{-1} ; 1\right]$ or $\left[2 * 0, \Sigma_{(2)} ; 1\right]$. In this case, $2(K+D) \sim 0$ and $K+D \nsim 0$.

COROLlary 1. Suppose that $q=1$. Then a relatively minimal pair $(S, D)$ is an Enriques pair if and only if $P_{2}[D]=1$ and $P_{3}[D]=0$.

THEOREM 3. Suppose that $q \geq 2$ and that $(S, D)$ is relatively minimal. If $\kappa[D]=1$, then $n=0$. If $\kappa[D]=2$, then $n \geq 4(q-1)$. Furthermore, we have the following table.

\begin{tabular}{c|c|c}
\hline class & $\kappa[D]$ & $\sigma(D)$ \\
\hline \hline I & $-\infty$ & 0 or 1 \\
\hline II & 0 & none \\
\hline II $\frac{1}{2}$ & 1 & 2 \\
\hline III & 2 & $\leq 2+n /(2 q-2)$ \\
\hline
\end{tabular}

The author expresses gratitude to Professors Shigeru Iitaka and Kazuo Akao for their valuable advices during the preparation of this paper. Moreover, the author would like to 
thank the referee for his very valuable suggestions which are essential to complete the final draft.

1.1. Elementary transformations and $\sharp$-minimal pairs. Let $A=\{z \mapsto \alpha z+$ $\beta ; \alpha \neq 0, \beta \in \mathbf{C}\}$ denote the 1-dimensional affine transformation group, which has a subgroup $\mathbf{C}^{*}$ consisting of non-zero complex numbers. A $\mathbf{P}^{1}$-bundle $X$ is said to be a $\mathbf{C}^{*}$-bundle or an affine-bundle if the structure group of $X$ is $\mathbf{C}^{*}$ or $A$, respectively.

By Atiyah [At], if $q=1$, then the degree of $\mathbf{C}^{*}$-bundle $X$ is non-negative and the degree of affine-bundle $X$ is zero or minus one, which is denoted by $A_{0}$ or $A_{-1}$, respectively. The $\mathbf{C}^{*}$-bundle with degree $b(>0)$ is isomorphic to each other. Note that $\mathbf{C}^{*}$-bundle $X$ of degree $b$ has two mutually disjoint sections $\Gamma_{0}$ and $\Gamma_{\infty}$ such that $\Gamma_{0}^{2}=b$ and $\Gamma_{\infty}^{2}=-b$. If $X$ is a direct product $S_{0}=\mathbf{P}^{1} \times B$, then there exist an infinite number of sections $\Gamma$ with $\Gamma^{2}=0$.

Let $\Phi: X \rightarrow B$ be the projection of the $\mathbf{P}^{1}$-bundle $X$. Elementary transformations of $\mathbf{P}^{1}$-bundles $X$ are defined as follows:

Take a point $p_{1}$ on $X$. Blowing up at $p_{1}$, we have a birational morphism $\mu: S_{1} \rightarrow X$. The fiber $F_{0}=\Phi^{-1}\left(\Phi\left(p_{1}\right)\right)$ has the proper inverse image $F_{0}^{\prime}$ by $\mu$ and the exceptional curve (of the first kind ) $E=\mu^{-1}\left(p_{1}\right)$. Then $F_{0}^{\prime 2}=-1$ and $F_{0}^{\prime}$ turns out to be an exceptional curve. Contracting $F_{0}^{\prime}$ into a non-singular point $p_{1}^{\prime}$, we have a non-singular surface $X^{\prime}$ and a proper birational morphism $\mu^{\prime}: S_{1} \rightarrow X^{\prime}$. Here $X^{\prime}$ is also a $\mathbf{P}^{1}$-bundle. The birational map $\mu^{\prime} \cdot \mu^{-1}$ is called an elementary transformation with center $p_{1}$.

Let $C$ be a curve on $X$. The proper image of $C$ by the birational map $\mu^{\prime} \cdot \mu^{-1}$ is denoted by $C^{\prime}$. The curve $C^{\prime}$ has the invariants $\sigma^{\prime}, e^{\prime}$ defined by $C^{\prime} \equiv \sigma^{\prime} \Gamma_{\infty}^{\prime}+e^{\prime} F^{\prime}$. Moreover, let $m_{1}^{\prime}$ denote the multiplicity of $C^{\prime}$ at $p_{1}^{\prime}$. It is easy to show that $\sigma^{\prime}=\sigma, e^{\prime}=e+\sigma-m_{1}$, if $p_{1} \in \Gamma_{\infty}$ and $\sigma^{\prime}=\sigma, e^{\prime}=e-m_{1}$, if $p_{1} \notin \Gamma_{\infty}$. Note that if $2 \leq \sigma<2 m_{1}$, then $m_{1}^{\prime}=\sigma-m_{1}<m_{1}$. Repeating such transformations if necessary, we may suppose $\sigma \geq 2 m_{1}$, when $\sigma \geq 2$.

Definition. The pair $(X, C)$ is said to be $\sharp$-minimal if $\sigma \geq 2 m_{1}$ (cf. I1).

Suppose that a relatively minimal pair $(S, D)$ satisfies $\sigma(D) \geq 2$. Then contracting exceptional curves successively, we have a $\mathbf{P}^{1}$-bundle $X$ and a birational morphism $\lambda: S \rightarrow$ $X$. The image of $D$ by $\lambda$ is denoted by a curve $C$. Assume that $C \equiv \sigma \Gamma_{\infty}+e F_{u}$. Then it is obviously that $\sigma(D)=\sigma$. From the previous argument, we infer the following proposition.

PROPOSITON 1. Assume that $(S, D)$ is a relatively minimal pair with $\sigma(D) \geq 2$. Then contracting exceptional curves successively and after a finite number of elementary transformations, $(S, D)$ is transformed into a $\sharp$-minimal pair $(X, C)$.

REMARK 1. Suppose that $(S, D)$ is a relatively minimal pair with $\kappa[D] \geq 0$. Then since $|m(K+D)| \neq \emptyset$, it follows that $m(K+D) \cdot F=m(\sigma(D)-2) \geq 0$ where $F$ denotes a general fiber of $\alpha: S \rightarrow B$. Hence, $\sigma(D) \geq 2$. Conversely, assume that $\sigma(D) \geq 2$. Then an addition formula of logarithmic Kodaira dimension by Kawamata $[\mathrm{K}]$ yields that $\kappa[D] \geq 0$. But here we shall study pairs more precisely and thus provide another proof of the result. 


\section{Case of ruled surfaces with irregularity 1.}

In this section, we suppose that $q=1$, i.e., $B$ is an elliptic curve.

2.1. Elementary proof of $\kappa[D] \geq 0$. Let $(X, C)$ be a $\sharp$-minimal pair and let $[\sigma *$ $\left.e, X ; m_{1}, m_{2}, \cdots, m_{r}\right]$ denote the type of $(X, C)$. Letting $\pi$ and $K_{0}$ be the virtual genus of $C$ and a canonical divisor of $X$, respectively, we have

$$
K_{0} \cdot C=\sigma b-2 e, \quad C^{2}=2 \sigma e-\sigma^{2} b, \quad \pi=\frac{(\sigma-1)(2 e-\sigma b)}{2}+1 .
$$

By blowing ups singular points on $C$ over $X$ successively, we have a birational morphism $\lambda: S \rightarrow X$, which gives rise to a shortest resolution of singularities of the embedded curve $C$ on $X$. Let $D$ denote the proper inverse image of $C$ by $\lambda$. In this case, the pair $(S, D)$ said to be a shortest model of $(X, C)$. We have the next.

Proposition 2. Let $(S, D)$ be a shortest model of $a \sharp$-minimal pair $(X, C)$. Then $\kappa[D] \geq 0$.

ProOF. By definition,

$$
K \sim \lambda^{*}\left(K_{0}\right)+\sum_{j=1}^{r} E_{j}, \quad D \sim \lambda^{*}(C)-\sum_{j=1}^{r} m_{j} E_{j},
$$

where the symbol $\sim$ means linear equivalence between divisors. The $E_{j}$ are exceptional curves derived from blowing up singular points $p_{j}$. For simplicity, the total inverse images of $E_{j}$ are denoted by the same symbols $E_{j}$. From $K_{0} \equiv-2 \Gamma_{\infty}-b F_{u}$ and $C \equiv \sigma \Gamma_{\infty}+e F_{u}$, we have

$$
\sigma K+2 D \sim \lambda^{*}\left(2 C+\sigma K_{0}\right)+\sum_{j=1}^{r}\left(\sigma-2 m_{j}\right) E_{j}, \quad \sigma K_{0}+2 C \equiv(2 e-b \sigma) F_{u} .
$$

LEMMA 1. Let $Y$ be a curve on a surface $X$ which is a $\mathbf{P}^{1}$-bundle over a curve. Then there exist $\alpha, \beta$ such that $Y \equiv \alpha \Gamma_{\infty}+\beta F_{u}$ where $\Gamma_{\infty}$ is the infinite section such that $\Gamma_{\infty}^{2}=$ $-b$. Suppose that $Y \neq F_{u}$ and $Y \neq \Gamma_{\infty}$. If $b \geq 0$ then $\alpha>0$ and $\beta \geq b \alpha$. If $b=-1$ then $\alpha>0$ and $\beta \geq-\alpha / 2$.

PROOF. See Proposition 2.21 of chapter V in [Ha].

By Lemma 1 , we have $2 e-b \sigma \geq 0$. Moreover, $2 e-b \sigma=0$ if and only if $(b, e)=(0,0)$ or $(b, e)=(-1,-\sigma / 2)$.

(i) Case $2 e-b \sigma>0$ : Then $\sigma K_{0}+2 C \equiv(2 e-b \sigma) F_{u}$ and $\mathcal{O}\left(\sigma K_{0}+2 C\right) \cong \Phi^{*}(\mathcal{L})$ for some invertible sheaf $\mathcal{L}$ on $C$ such that $\operatorname{deg}(\mathcal{L})=2 e-b \sigma$. By the Riemann-Roch theorem on $B$,

$$
\operatorname{dim} H^{0}\left(X, \mathcal{O}\left(\sigma K_{0}+2 C\right)\right)=\operatorname{dim} H^{0}(B, \mathcal{L})=\operatorname{deg}(\mathcal{L})=2 e-b \sigma>0 .
$$

Hence, $\left|\sigma K_{0}+2 C\right| \neq \phi$, which induces $|\sigma K+2 D| \neq \phi$, too. Thus,

$$
0 \leq \kappa(\sigma K+2 D, S) \leq \kappa(\sigma(K+D), S)=\kappa(K+D, S)=\kappa[D] .
$$


(ii) Case $2 e-b \sigma=0$ : Then $(b, e)=(0,0)$ or $(b, e)=(-1,-\sigma / 2)$. In both cases, we can verify $C^{2}=0$ and $C \cdot K_{0}=0$ and hence $\pi(C)=1$. However, since $\sigma \geq 2$ is assumed, it follows that $\Phi(C)=B$ and so $C$ cannot be a rational curve. Hence, $C$ is a non-singular elliptic curve, i.e., $S=X$ and $D=C$. The restriction of $\Phi$ to $C$ turns out to be an étale morphism $f: C \rightarrow B$. From $\Phi: X \rightarrow B$ and $f: C \rightarrow B$, we obtain a fiber product $W=X \times{ }_{B} C$, which has projections $\bar{f}: W \rightarrow X$ and $\bar{\Phi}: W \rightarrow C$. To complete the proof of Proposition 2, we prepare the following proposition.

Proposition 3. W is isomorphic to the product $C \times \mathbf{P}^{1}$ except for the case $b=-1$ and $\sigma=2$.

Proof. From $W=\{(x, p) \in X \times C \mid \Phi(x)=f(p)\}$, it follows that $\bar{\Phi}^{-1}(p)=$ $\Phi^{-1}(f(p)) \cong \mathbf{P}^{1}$ for any $p \in C$. Hence, $W$ is a $\mathbf{P}^{1}$-bundle over $C$. Take a general point $p_{1} \in$ $C$. Then $f^{-1}\left(f\left(p_{1}\right)\right)=\left\{p_{1}, p_{2}, \cdots, p_{\sigma}\right\}$. The curves $\Gamma_{j}=\left\{\left(p_{1}, p_{j}\right) \in C \times C \mid p_{1} \in C\right\}$ are sections of $\bar{\Phi}: W \rightarrow C$ and $\Gamma_{i} \cap \Gamma_{j}=\emptyset$ for $i \neq j$. Note that if $b=0$ and $\sigma=2$, then $\bar{f}^{-1}\left(\Gamma_{\infty}\right)$ is a section and $\Gamma_{i} \cap \bar{f}^{-1}\left(\Gamma_{\infty}\right)=\emptyset$ for any $i$. Hence $W$ has at least three sections such that any pair of sections has no common points, which implies that $W$ is isomorphic to the product $C \times \mathbf{P}^{1}$.

We shall proceed with the proof of Proposition 2. By Proposition 3, $\bar{f}^{*}(C) \sim \sigma \bar{C}$ and $K_{W} \sim-2 \bar{C}$ where $\bar{C}$ is a copy of $C$. Since $K_{W}+\bar{f}^{*} C \sim(\sigma-2) \bar{C}$, we have

$$
\kappa[D]=\kappa\left(X, K_{X}+C\right)=\kappa\left(W, K_{W}+\bar{f}^{*} C\right)=\kappa\left(C \times \mathbf{P}^{1},(\sigma-2) \bar{C}\right) \geq 0 .
$$

In the case when $b=-1$ and $\sigma=2$, a pair of type $\left[2 * 0, \Sigma_{(2)} ; 1\right]$ is transformed into a pair of type $\left[2 *-1, A_{-1} ; 1\right]$ by an elementary transformation. Thus, $\kappa[D] \geq 0$. This completes the proof of Proposition 2.

\subsection{Properties of relatively minimal pairs.}

Proposition 4. Let $(S, D)$ be a relatively minimal pair with $\kappa[D] \geq 0$. Then $K+D$ is nef.

Proof. Note that $\sigma(D) \geq 2$, whenever $\kappa[D] \geq 0$ by Remark 1 . Then since $D$ is a curve over $B, g(D) \geq q=g(B) \geq 1$. Suppose that there exists an irreducible curve $A$ on $S$ such that $(K+D) \cdot A<0$. If $A=D$, then $(K+D) \cdot D=2 g(D)-2<0$. Thus, $g(D)=0$, which contradicts the fact that $g(D) \geq 1$. Therefore, $D \neq A$ and so $A \cdot D \geq 0$. Since $A^{2}<0$ and $K \cdot A<-D \cdot A \leq 0$, it follows that $A$ is an exceptional curve and moreover, $-1<-D \cdot A \leq 0$, which implies that $D \cdot A=0$. However, this contradicts the hypothesis that $(S, D)$ is relatively minimal.

PROPOSITION 5. Let $(S, D)$ be a shortest model of a $\sharp$-minimal pair $(X, C)$. Then $(S, D)$ is relatively minimal.

Proof. Suppose that $(S, D)$ is not relatively minimal. Then $S \neq X$ and there exists an exceptional curve $A$ such that $A \cdot D=1$ or 0 . Letting $A_{0}=\mu(A)$, we have

$$
2-\sigma \geq(\sigma K+2 D) \cdot A \geq\left(\sigma K_{0}+2 C\right) \cdot A_{0}=(2 e-b \sigma) F_{u} \cdot A_{0} \geq 0 .
$$


Hence, $\sigma=2$, which implies that $D=C$ and $S=X$.

LEMMA 2. $\kappa[D]=2$ if and only if $(K+D)^{2}>0$.

PROOF. By Corollary 14.18 in [B] or Proposition 1 in [Sa2], we have the result.

2.3. Proof of Theorem 1. First we note that $\kappa[D]=-\infty$ if and only if $\sigma(D)=0$ or 1 by Remark 1 .

Second, suppose that $\kappa[D] \geq 0$. Then $\sigma(D) \geq 2$. By Lemma 2, $\kappa[D]=0$ or 1 if and only if $(K+D)^{2}=0$. Assume that $(K+D)^{2}=0$. Since $K+D \equiv(\sigma-2) \Gamma_{\infty}+(e-$ b) $F_{u}-\sum_{j=1}^{r}\left(m_{j}-1\right) E_{j}$, we obtain

$$
(K+D)^{2}=(\sigma-2)(2 e-\sigma b)-\sum_{j=1}^{r}\left(m_{j}-1\right)^{2}
$$

Thus by hypothesis,

$$
(\sigma-2)(2 e-\sigma b)=\sum_{j=1}^{r}\left(m_{j}-1\right)^{2}
$$

By the adjunction formula, letting $g=g(D)$,

$$
0 \leq 2 g-2=D^{2}+K \cdot D=(D+K) \cdot D=(\sigma-1)(2 e-b \sigma)-\sum_{j=1}^{r} m_{j}\left(m_{j}-1\right) \text {. }
$$

Hence,

$$
(\sigma-2)(2 e-\sigma b) \geq \sum_{j=1}^{r} m_{j}\left(m_{j}-1\right)-(2 e-\sigma b) .
$$

From (2) and (3), it follows that

$$
\sum_{j=1}^{r}\left(m_{j}-1\right)^{2}=(\sigma-2)(2 e-\sigma b) \geq \sum_{j=1}^{r} m_{j}\left(m_{j}-1\right)-(2 e-\sigma b) .
$$

Hence, $2 e-\sigma b \geq \sum_{j=1}^{r}\left(m_{j}-1\right)$. Multiplying both sides by $\sigma-2$,

$$
(\sigma-2)(2 e-\sigma b) \geq(\sigma-2) \sum_{j=1}^{r}\left(m_{j}-1\right) .
$$

Recalling the equality (2), we obtain

$$
\sum_{j=1}^{r}\left(m_{j}-1\right)^{2} \geq(\sigma-2) \sum_{j=1}^{r}\left(m_{j}-1\right) .
$$

Hence,

$$
\sum_{j=1}^{r}\left(m_{j}-1\right)\left(m_{j}+1-\sigma\right) \geq 0 .
$$


Since $\sigma \geq 2 m_{j}$, it follows that $m_{j}+1-\sigma \leq 1-m_{j}$. Hence $\sum_{j=1}^{r}\left(m_{j}-1\right)\left(1-m_{j}\right) \geq 0$; thus, i.e., $r=0$ and so $S=X$ and $D=C$. By the equality (2),

$$
(\sigma-2)(2 e-\sigma b)=0 .
$$

Hence, we obtain either $\sigma=2$ or $2 e-\sigma b=0$.

Thus we have two cases to examine, separately.

(I) case $2 e-\sigma b=0$ : Then $(b, e)=(0,0)$ or $(b, e)=(-1,-\sigma / 2)$.

Let $\kappa^{-1}(S)$ denote $\kappa(S,-K)$, which means the anti-Kodaira dimension of $S$. Define $S_{0}$ to be the product of $\mathbf{P}^{1}$ and an elliptic curve $E$.

LEMMA 3. If there exists an étale morphism $\varphi: S_{0} \rightarrow X$, then $X$ has a structure of an elliptic surface.

Proof. Since $\varphi$ is an étale morphism, $\kappa^{-1}\left(S_{0}\right)=\kappa^{-1}(X)$. Thus, $\kappa^{-1}(X)=$ $\kappa^{-1}\left(S_{0}\right)=1$, which implies that $X$ is an elliptic surface.

Lemma 4. Suppose that $X$ is an elliptic ruled surface. Then $X$ is isomorphic to one of the following:

$$
\text { (1) } \mathbf{P}^{1} \times E, \quad \text { (2) } \Sigma_{(k)} \text { (3) } A_{-1} \text {. }
$$

Here $\Sigma_{(k)}$ has a general elliptic fiber which is numerically equivalent to $k \Gamma_{\infty}$.

ProOF. See Theorem 5 of [Sw].

Employing the notation in the proof of Proposition 3, since $W \cong \mathbf{P}^{1} \times C$ and $\bar{f}: W \rightarrow X$ is an étale morphism, $X$ is an elliptic surface by Lemma 3. On the other hand, since ( $b=$ $0, e=0)$ or $(b=-1, e=-\sigma / 2)$, it follows that $C^{2}=0$ and $K_{0} \cdot C=0$. Hence, $C$ is an elliptic curve which is a fiber of the elliptic fiber space $\psi: X \rightarrow \mathbf{P}^{1}$. If $b=0$, then since $C$ is a general elliptic fiber and $C \equiv \sigma \Gamma_{\infty}$, we obtain $X \supsetneqq \mathbf{P}^{1} \times C$. Hence, $X$ is isomorphic to either $\Sigma_{(\sigma)}$ or $A_{-1}$.

We shall examine the following cases, separately.

(I-1) $(b, e)=(0,0)$ : Then $X=\Sigma_{(\sigma)}$. From the canonical bundle formula by Kodaira [Ko], $K=K_{0} \sim \psi^{*}(-2 P)+(\sigma-1) F_{1}+(\sigma-1) F_{2}$ and $D=C \sim \psi^{*}(P)$, where $P$ is a point on $\mathbf{P}^{1}$ and $\sigma F_{i} \sim \psi^{*}(P)$. Hence,

$$
\sigma K_{0}+2 C \sim 0 .
$$

In the case of $\sigma=2$, we have $2\left(K_{0}+C\right) \sim 0$. Hence, $P_{2 m}[D]=1$ and $P_{2 m-1}[D]=0$ for any $m>0$. In this case, we have $\kappa[D]=0$.

Suppose that $\sigma \geq 3$. For a positive integer $m$, let $s$ be the quotient of $m(\sigma-1)$ by $\sigma$ with the remainder $\rho$, i.e., $m(\sigma-1)=s \sigma+\rho$. Then for $i=1,2$,

$$
m(\sigma-1) F_{i} \sim \psi^{*}(s P)+\rho F_{i} .
$$

Thus, it follows that

$$
m\left(K_{0}+C\right) \sim \psi^{*}((-m+2 s) P)+\rho\left(F_{1}+F_{2}\right) .
$$


If $m=1$, then $s=0$ and so $P_{1}[D]=0$. If $m \geq 2$, then $2 s-m \geq 2[2 m / 3]-m \geq 0$. Hence, $P_{m}[D]=1+2 s-m \geq 1$ for any $m \geq 2$. If $m \geq 3$, then $2 s-m>0$. Thus, $\kappa[D]=1$.

(I-2) $(b, e)=(-1,-\sigma / 2)$ where $\sigma \geq 3$ : Since $X=A_{-1}$ and since $D=C$ is a general elliptic fiber, we have $\sigma=4$. We see that $K=K_{0} \sim \psi^{*}(-2 P)+F_{1}+F_{2}+F_{3}$ and $D=C \sim \psi^{*}(P)$ where $2 F_{i} \sim \psi^{*}(P)$. For a positive integer $m$, let $s$ be the quotient of $m$ by 2 with the remainder $\rho$. Then it follows that

$$
m\left(K_{0}+C\right) \sim \psi^{*}((-m+3 s) P)+\rho\left(F_{1}+F_{2}+F_{3}\right)
$$

If $m=1$, then $s=0$ and so $P_{1}[D]=0$. If $m \geq 2$, then $3 s-m \geq 3[m / 2]-m \geq 0$. Hence, $P_{m}[D]=1+3 s-m$ for any $m>0$. In this case, we have $\kappa[D]=1$.

(II) case $\sigma=2$ and $2 e-\sigma b \neq 0$ : We see that if $b \geq 1$, then $e \geq \sigma b$ and if $b=0$, then $e \geq 1$ and if $b=-1$, then $e \geq 0$. Therefore, $K_{0}+C \equiv(e-b) F_{u}$ where $e-b$ is positive. Moreover, $\mathcal{O}\left(K_{0}+C\right) \cong \Phi^{*}(\mathcal{L})$ for some invertible sheaf $\mathcal{L}$ on $C$ such that $\operatorname{deg}(\mathcal{L})=e-b$. By the Riemann-Roch theorem on $B$,

$$
\operatorname{dim} H^{0}\left(X, \mathcal{O}\left(m\left(K_{0}+C\right)\right)\right)=\operatorname{dim} H^{0}\left(B, \mathcal{L}^{\otimes m}\right)=\operatorname{deg}\left(\mathcal{L}^{\otimes m}\right)=m(e-b)>0 .
$$

Hence, $P_{m}[C]=m(e-b)$ for $m \geq 1$. In this case, we have $\kappa[D]=1$. Moreover, $g(D)=$ $\left(C+K_{0}\right) \cdot C / 2+1=e-b+1 \geq 2$. This completes the proofs of I, II and II $\frac{1}{2}$ in Theorem 1 .

LEMMA 5. If $\kappa[D]=2$ and $m \geq 2$ then

$$
P_{m}[D]=\frac{m(m-1)}{2}(K+D)^{2}+\frac{m}{2}(K+D) \cdot D+\max \{0,2-g(D)\} \geq 2 .
$$

Proof. By Lemma (4.2) in [Sa1], $\operatorname{dim} H^{1}(S, \mathcal{O}(m(K+D)))=\operatorname{dim} H^{1}(D$, $\left.\mathcal{O}\left(m K_{D}\right)\right)=1$ or 0 according to $g(D)=1$ or $g(D) \geq 2$, respectively. Hence we have the result by the Riemann-Roch theorem.

Therefore, the proof of Theorem 1 is complete.

2.4. Proof of Theorem 2. By $\kappa[D]=2$, we obtain $(K+D)^{2}>0$ and so $D^{2}<$ $4 g-4-r$ where $g$ denotes the genus of the curve $D$. Hence, $0 \leq r<4 g-D^{2}-4=n$.

(1) Case $m_{1} \leq 2$. Then $D^{2}=\sigma(2 e-\sigma b)-4 r$ and $4 g=2(\sigma-1)(2 e-\sigma b)+4-4 r$. These imply that

$$
1 \leq n=4 g-D^{2}-4=(2 e-\sigma b)(\sigma-2)
$$

Thus, $\sigma-2 \leq n$; hence $\sigma \leq n+2 \leq n(n+2)$. 
(2) Case $m_{1} \geq 3$. Then $\sigma \geq 6$. Since $D^{2}=C^{2}-\sum_{j=1}^{r} m_{j}^{2}$ and $g=\pi-$ $\sum_{j=1}^{r} m_{j}\left(m_{j}-1\right) / 2$, we obtain

$$
\begin{aligned}
& \sum_{j=1}^{r} m_{j}=C^{2}-2 \pi-D^{2}+2 g=n+t-2(g-1) \\
& \sum_{j=1}^{r} m_{j}^{2}=C^{2}-D^{2}=\sigma t+n-4(g-1)
\end{aligned}
$$

where $t=2 e-\sigma b \geq 0$. From (8) and (9), it follows that

$$
\begin{aligned}
0 & \leq\left(\sum_{j=1}^{r} m_{j}\right)^{2}-\sum_{j=1}^{r} m_{j}^{2} \\
& =(n+t)^{2}-4(n+t)(g-1)+4(g-1)^{2}-\sigma t-n+4(g-1) \\
& =(n+t)^{2}-\sigma t-n-4(n+t-g)(g-1) \\
& \leq(n+t)^{2}-\sigma t-n-4(g-2)(g-1) \\
& \leq(n+t)^{2}-\sigma t-n .
\end{aligned}
$$

Hence,

$$
\sigma t \leq(n+t)^{2}-n
$$

If $t=0$, then from (8) and (9), it follows that

$$
\sum_{j=1}^{r} m_{j}\left(m_{j}-1\right)=-2(g-1) .
$$

Hence, we have $g=1$ and $r=0$. This contradicts the hypothesis $m_{1} \geq 3$. Thus, $t>0$ and so

$$
\sigma \leq \frac{(n+t)^{2}-n}{t}
$$

On the other hand, from (8) and (9), it follows that

$$
0 \leq m_{1} \sum_{j=1}^{r} m_{j}-\sum_{j=1}^{r} m_{j}^{2}=\left(m_{1}-1\right) n-\left(\sigma-m_{1}\right) t-2(g-1)\left(m_{1}-2\right) .
$$

Hence,

$$
\left(\sigma-m_{1}\right) t \leq\left(m_{1}-1\right) n-2(g-1)\left(m_{1}-2\right) .
$$

Since $\sigma \geq 2 m_{1}$ and $g \geq 1$, it follows that $t<n$.

From $t<n$ and the equality (11), we obtain

$$
\sigma=\sigma(D) \leq \frac{(n+t)^{2}-n}{t}<n(n+2) .
$$

This completes the proof of the first part of Theorem 2 . 
Proposition 6. Let $(S, D)$ be a relatively minimal pair with $\kappa[D]=2$ and $n=1$. Then the type of $(S, D)$ is $\left[3 *-1, A_{-1} ; 1\right]$ and $g(D)=2, D^{2}=3$. Furthermore, $P_{m}[D]=$ $m(m+1) / 2$ for any $m \geq 1$.

To prove Proposition 6, we prepare following lemmas.

Lemma 6. Let $A$ be a divisor on $A_{-1}$. Suppose that $A \equiv \alpha \Gamma_{\infty}+\beta F$. Then $A$ is ample if and only if $\alpha>0, \beta>-\alpha / 2$.

PROOF. See Proposition 2.21 of p. 382 of [Ha].

LEMMA 7. $P_{1}[D]=g(D)-1+h(D)$, where $h(D)$ denotes $\operatorname{dim} H^{1}(S, \mathcal{O}(-D))$.

Proof. By the Riemann-Roch theorem, Serre duality and the adjunction formula,

$P_{1}[D]-h(D)=\operatorname{dim} H^{0}(S, \mathcal{O}(K+D))-\operatorname{dim} H^{1}(S, \mathcal{O}(K+D))=\frac{(K+D) \cdot D}{2}=g(D)-1$.

Proof of Proposition 6. By the equality (7), we have $(2 e-\sigma b)(\sigma-2)=1$; hence, $2 e-\sigma b=1$ and $\sigma=3$. Thus, the type of the pair is $\left[3 *-1, A_{-1} ; 1\right]$. In this case, $g(D)=2$ and $D^{2}=3$.

Moreover, let us consider a linear system $\left|\bar{F}+\Gamma_{\infty}\right|$ where $\bar{F}$ is a double fiber of the elliptic surface $A_{-1}$, which satisfies $\bar{F} \equiv 2 \Gamma_{\infty}-F_{u}$. Let $D$ be a general element of $\left|\bar{F}+\Gamma_{\infty}\right|$. Then $D \equiv 3 \Gamma_{\infty}-F_{u}$. By Lemma $6, D$ is an ample divisor. Therefore, by the Kodaira vanishing theorem, $\operatorname{dim} H^{1}\left(A_{-1}, \mathcal{O}\left(K_{0}-D\right)\right)=0$. By the Riemann-Roch theorem, we have $\operatorname{dim} H^{0}\left(A_{-1}, \mathcal{O}(D)\right)=2$.

On the other hand, by Lemma 6 and $\Gamma_{\infty}-K_{0} \equiv 3 \Gamma_{\infty}-F_{u}, \Gamma_{\infty}-K_{0}$ is an ample divisor. Hence, by the Kodaira vanishing theorem, $\operatorname{dim} H^{1}\left(A_{-1}, \mathcal{O}\left(\Gamma_{\infty}-K_{0}+K_{0}\right)\right)=0$. Hence, $\operatorname{dim} H^{0}\left(A_{-1}, \mathcal{O}\left(\Gamma_{\infty}\right)\right)=1$ by the Riemann-Roch theorem. Furthermore, since $\mathcal{O}(2 \bar{F}) \sim$ $\psi^{*} \mathcal{O}(1)$, it follows that $\operatorname{dim} H^{0}\left(A_{-1}, \mathcal{O}(\bar{F})\right)=1$. From $\operatorname{dim} H^{0}\left(A_{-1}, \mathcal{O}\left(\bar{F}+\Gamma_{\infty}\right)\right)=2$, $\left|\bar{F}+\Gamma_{\infty}\right|$ is free from fixed components. Since $D \in\left|\bar{F}+\Gamma_{\infty}\right|$ and $D^{2}=3, D$ is irreducible by a theorem of Bertini.

By $\operatorname{dim} H^{1}\left(A_{-1}, \mathcal{O}(-D)\right)=0$ and Lemma 7 , it follows that $P_{1}[D]=2-1+0=1$. Moreover, Since $g(D)=2,(K+D)^{2}=\Gamma_{\infty}^{2}=1$ and $D \cdot(K+D)=\left(3 \Gamma_{\infty}-F_{u}\right) \cdot \Gamma_{\infty}=2$, we obtain $P_{m}[D]=m(m+1) / 2$ for all $m \geq 2$ by Lemma 5 .

This completes the proof of Proposition 6.

\subsection{Minimal pairs.}

Lemma 8. Suppose that $\alpha(K+D)+\tau K$ is neffor some $\alpha \geq 1$ and $\tau>0$. Then the pair $(S, D)$ is minimal.

PROOF. See Proposition 4 of [I1].

PROPOSITION 7. Suppose that every relatively minimal pair $(S, D)$ with $\kappa[D] \geq 1$ is minimal unless the type of $(S, D)$ is $[2 * e, X ; 1]$. In any case, $(K+D)^{2}$ is a birational invariant for pairs. 
Proof. Suppose that $\kappa[D]=1$ and that the type of $(S, D)$ is not $[2 * e, X ; 1]$. (Case A) Assume that the type is $\left[\sigma * 0, \Sigma_{(\sigma)} ; 1\right]$ where $\sigma \geq 3$. Then $\sigma K+2 D \sim 0$ by (6). Hence, $2(K+D)+(\sigma-2) K \sim 0$, which is clearly nef. (Case B) Assume that the type is $\left[4 *(-2), A_{-1} ; 1\right]$. Then by the canonical bundle formula of Kodaira [Ko], $2 K+D \sim 0$, which is clearly nef.

If $\kappa[D]=2$, then by a similar argument to the proof of Proposition 3 of [I1], we see that $(m-1)(K+D)+K$ is nef for sufficiently large $m$. Hence by Lemma 8 , we complete the proof.

\section{Case of ruled surfaces with irregularity $q \geq 2$.}

In this section, we shall study the birational structure of pairs $(S, D)$ with irregularity $q \geq 2$.

Let $(X, C)$ be a $\sharp$-minimal pair with type $\left[\sigma * e, X ; m_{1}, \cdots, m_{r}\right]$ where $X$ is a $\mathbf{P}^{1}$-bundle with irregularity $q \geq 2$. Let $(S, D)$ be a shortest model of a $(X, C)$. Employing the same notation as in section 2 , we have

$$
\sigma K+2 D \equiv \sigma K_{0}+2 C+\sum_{j=1}^{r}\left(\sigma-2 m_{j}\right) E_{j}, \quad \sigma K_{0}+2 C \equiv(2 e-\sigma b+2 \sigma(q-1)) F
$$

Since $C$ is an irreducible curve, it follows that $2 e \geq \sigma b$. Moreover, $e \geq \sigma b$ if $b \geq 0$ (cf. [Ha]). $\sigma K_{0}+2 C$ is written as a pull back of a divisor $\Theta$ on $B$. Then

$$
\begin{aligned}
\operatorname{deg} \Theta= & 2 e-\sigma b+2 \sigma(q-1) \geq 2 \sigma(q-1) \geq 4(q-1) \geq 2 q, \\
& \operatorname{dim}\left|\sigma K_{0}+2 C\right|=\operatorname{dim}|\Theta|=\operatorname{deg} \Theta-q \geq 2 .
\end{aligned}
$$

Hence, $\kappa[D] \geq 1$. Moreover, $K+D$ is nef and $(S, D)$ is a relatively minimal pair by a similar argument to the proofs of Propositions 4 and 5. Furthermore, by following the argument of the proof of Proposition 7, we see that any relatively minimal pair $(S, D)$ is minimal whenever $\kappa[D]=2$.

3.1. Proof of Theorem 3. (1) Suppose that $\kappa[D]=1$. Since $K+D$ is nef, $\kappa[D]=$ 1 if and only if $(K+D)^{2}=0$. Then from $K_{0}+C \equiv(\sigma-2) \Gamma_{\infty}+(e-b+2 q-2) F_{u}$ and

$$
0=(K+D)^{2}=\left(K_{0}+C\right)^{2}-\sum_{j=1}^{r}\left(m_{j}-1\right)^{2}
$$

it follows that

$$
(\sigma-2)(2 e-\sigma b+4 q-4)=\sum_{j=1}^{r}\left(m_{j}-1\right)^{2} .
$$

On the other hand, since

$$
2 q \leq 2 g=(\sigma-1)(2 e-\sigma b+2 q-2)+2 q-\sum_{j=1}^{r} m_{j}\left(m_{j}-1\right)
$$


it follows that

$$
(\sigma-2)(2 e-\sigma b+4 q-4)-2(\sigma-1)(q-1)+(2 e-\sigma b+4 q-4) \geq \sum_{j=1}^{r} m_{j}\left(m_{j}-1\right) .
$$

Hence, from

$$
\sum_{j}^{r}\left(m_{j}-1\right)^{2}-2(\sigma-1)(q-1) \geq \sum_{j=1}^{r} m_{j}\left(m_{j}-1\right)-(2 e-\sigma b+4 q-4),
$$

we infer

$$
\sum_{j=1}^{r}\left(m_{j}-1\right)+2(\sigma-1)(q-1) \leq 2 e-\sigma b+4 q-4
$$

By $\sharp$-minimality,

$$
2 \sum_{j=1}^{r}\left(m_{j}-1\right)^{2} \leq(\sigma-2) \sum_{j=1}^{r}\left(m_{j}-1\right)
$$

By (12) and (13),

$$
2 \sum_{j=1}^{r}\left(m_{j}-1\right)^{2}+2(\sigma-2)(\sigma-1)(q-1) \leq \sum_{j=1}^{r}\left(m_{j}-1\right)^{2} .
$$

Thus, $\sigma=\sigma(D)=2$ and $r=0$, which implies $n=0$. In this case, it follows that $\kappa[D]=1$.

(2) Suppose that $\kappa[D]=2$. Then $\sigma=\sigma(D) \geq 3$.

(2-1) Case $m_{1} \leq 2$. By $D^{2}-4 g=C^{2}-4 \pi=4-8 q-n$, we have

$$
(\sigma-2)(t+4 q-4)=n
$$

where $t=2 e-\sigma b \geq 0$ and $g$ denotes the genus of a curve $D$. Since $t \geq 0$ and $\sigma \geq 3$, it follows that $n \geq 4(\sigma-2)(q-1) \geq 4(q-1)$. Moreover, from $4 q-4 \neq 0$, we infer that $\sigma(D)=\sigma \leq 2+n /(4 q-4)<2+n /(2 q-2)$.

(2-2) Case $m_{1} \geq 3$. Then $\sigma \geq 6$ and furthermore,

$$
D^{2}=\sigma t-\sum_{j=1}^{r} m_{j}^{2}, \quad 4 g=2(\sigma-1)(t+2 q-2)+4 q-2 \sum_{j=1}^{r} m_{j}\left(m_{j}-1\right) .
$$

From this, we have

$$
\begin{aligned}
& \sum_{j=1}^{r} m_{j}=n-2(\sigma-3)(q-1)+t-2(g-q), \\
& \sum_{j=1}^{r} m_{j}^{2}=\sigma t+n+4(q-1)-4(g-q) .
\end{aligned}
$$


Hence,

$$
\begin{aligned}
0 & \leq \sigma \sum_{j=1}^{r} m_{j}-\sum_{j=1}^{r} m_{j}^{2} \\
& =\sigma n-2 \sigma(\sigma-3)(q-1)-2 \sigma(g-q)-n-4(q-1)+4(g-q) \\
& =(\sigma-1) n-2(\sigma-1)(\sigma-2)(q-1)-2(\sigma-2)(g-q) .
\end{aligned}
$$

Therefore, by $g \geq q$ and $\sigma \geq 3$,

$$
2 \sigma(q-1) \leq n+4(q-1)
$$

and so $\sigma(D)=\sigma \leq 2+n /(2 q-2)$. Recalling $\sigma \geq 6$, we have $n \geq 8(q-1)>4(q-1)$. This completes the proof of Theorem 3 .

\section{References}

[At] M. F. ATıYAh, Vector bundles over an elliptic curve, Proc. London Math. Soc. (3) 7 (1957), 414-452.

[B] L. BĂDESCU, Algebraic Surfaces, Universitext, Springer.

[Ha] R. HARTSHORne, Algebraic Geometry, Springer (1977).

[I1] S. IITAKA, On irreducible plane curves, Saitama Math. J. 1 (1983), 47-63.

[I2] S. IItAKA, Birational Geometry of Plane Curves, Tokyo J. Math. 22 (1999), 289-321.

[K] Y. KAWAmATA, Addition formula of logarithmic Kodaira dimensions for algebraic fiber spaces of relative dimension one, Proc. Int. Symp. Algebraic Geometry, Kyoto 1978, Kinokuniya, 207-217.

[Ko] K. KodAIRA, On the structure of compact complex analytic surfaces, I, American Journal of Mathematics, 86 (1964).

[Sa1] F. SAKAI, Semi-stable curve of algebraic surfaces and logarithmic pluricanonical maps, Math. Ann 254 (1980), 89-120.

[Sa2] F. SAKAI, D-dimensions of algebraic surfaces and numerically effective divisors, Compositio Math. 48 (1983).

[Sw] T. SuwA, On ruled surfaces of genus 1, J. Math. Soc. Japan 21 (1969), 291-311.

Present Address:

Tsuyama National College of Technology,

NUMA, TSUYAMA-CITY, OKAYAMA, 708-8509 JAPAN.

e-mail:matsuda@tsuyama-ct.ac.jp 